\title{
Foreword
}

\section{The National Cancer Institute Early Detection Research Network: 5th Scientific Workshop - Biomarkers-at-a-Crossroads}

\author{
Christos Patriotis* and Sudhir Srivastava \\ Cancer Biomarkers Research Group, National Cancer Institute, Bethesda, MD, USA
}

Over the past three decades a remarkable progress has been made toward understanding the molecular determinants of cancer and the development of new diagnostic and therapeutic strategies for the management of the disease. Cancer, however, remains the second leading cause of death, which is mainly due to the increasing incidence of cancer worldwide.

Major scientific and technological breakthroughs in the areas of genomics, proteomics, metabolomics, nanotechnology, molecular imaging and bioinformatics have created a realistic opportunity for the development of molecular biomarkers that are critical for population screening and the early detection of cancers, for better cancer diagnosis and prognosis, and for consideration of appropriate treatment modalities. However, we are still hampered by the heterogeneity of cancer at the molecular and histopathologic levels. Although several prognostic indicators based on clinical and histopathologic variables, such as tumor size, lymph node status and hormone receptor status are used in the clinic, these fail to determine the specific risk for recurrence and/or response to treatment. Molecular profiling is providing clues as to the heterogeneity and subtypes of cancers and being exploited towards identifying dis-

*Corresponding author: Christos Patriotis, Ph.D., Cancer Biomarkers Research Group, National Institutes of Health, Executive Plaza North, Room 3144, 6130 Executive Boulevard, Rockville, MD 20852-7346, USA. Tel.: +1 301435 1594; Fax: +1 301402 8990; E-mail: patriotisc@mail.nih.gov. ease subtypes. Two such prognostic profiles using microarray approaches have been developed that provide specific information about breast cancer prognosis: 1) the Amsterdam 70-gene profile, popularly known as Mammaprint has been approved by FDA for its ability to predict prognosis regardless of lymph node involvement and acts as an independent risk factor for distant metastasis; and 2) the 21-gene recurrence score indicator, popularly known as OncotypeDx, which provides reliable and reproducible information on the disease recurrence and therapeutic response.

An exciting emerging field in cancer research and diagnosis is based on the concept of stem/progenitor cells as being the primary targets of neoplastic transformation - "tumor stem cells" (TSC). It is believed that similar to normal tissue organization, tumor progression is driven by the TSC component. Presumably, that initial deregulation of stem cell growth and proliferation leads to a significant expansion of this progenitor cell population, which may harbor certain mutations that promote the acquisition of further oncogenic events and transformation. Hence, this new TSC concept and stem cell population expansion early on during neoplasia may be applied to the development of new methods for cancer risk assessment, early detection, prediction of disease progression and response to therapy.

MicroRNAs are small, non-coding functional RNAs, which play an intricate role in the regulation of translation of many genes, most notably oncogenes, by binding to the 3'-UTR of corresponding mRNA transcripts. 
Distinct microRNA expression patterns have been identified in association with a variety of cancers, including pancreatic, colon and lung. Hence, the deregulation of expression of specific microRNAs could be utilized for the development of biomarkers for early detection, risk assessment, diagnosis and prediction of disease progression, as well as targets for cancer therapy.

Many candidate biomarkers have been discovered to date, however, only few are available in the clinic for the early detection of cancer. The translation of molecular biomarkers from the research laboratory to the clinical setting is a lengthy and difficult process. For each candidate biomarker it is first necessary that a clinical test with high analytical sensitivity and accuracy to be first developed and optimized, and the performance of the test be then evaluated in large cohorts of unbiasly selected human biological specimens obtained by noninvasive means.

Over the past eight years, the National Cancer Institute's Early Detection Research Network (EDRN) has fostered a highly collaborative, multidisciplinary research venue for the discovery, development and clinical validation of biomarkers for risk assessment and the early detection of cancer. Since its inception, EDRN organizes every other year a scientific workshop at which EDRN- and non-EDRN investigators from the academia, the pharmaceutical industry and the NCI, as well as cancer advocates come together to present and discuss the most recent scientific and technological achievements in the field of cancer biomarker development and early cancer detection. The workshops have also been critical for the development of novel ideas and new collaborations between EDRN- and non-EDRN investigators, clinicians and technologists. The 5th EDRN Scientific Workshop - Biomarkersat-a-Crossroads, sponsored by the Cancer Biomarkers
Research Group, National Cancer Institute, took place on March 17-19, 2008 in Bethesda, MD. More than 400 scientists and cancer advocates, from the US and abroad, participated at the workshop. The keynote lectures and oral presentations included in six separate sessions, and the more than 90 abstracts presented at the poster session highlighted the new trends and achievements in biomarker discovery and development, novel technological and bioinformatics approaches implemented in the field, as well as some of the challenges of translating new biomarkers from the bench to the clinic. The abstracts of the presentations at the workshop are published in this special issue of Cancer Biomarkers.

Biomarkers are poised to aid in the quest of companion diagnostics. Such biomarkers are expected to provide further confirmatory information on the presence and molecular characteristics of cancer with higher precision and sensitivity such that they allow different types of cancers that affect the same organ to be distinguished. Such information can enable critical decisions, such as surgical removal of vital organs and/or preventive care, decisions related to suitable chemotherapy and other modalities, and for patient follow-up. For example, the clinical outcome and the therapeutic course of an individual patient with breast cancer are customarily uncertain due to the vastly heterogeneous nature of the disease and its diverse clinical outcomes.

The authors would like to extend their sincere appreciation to the members of the 5th EDRN Scientific Workshop Organizing Committee, Drs. S. Belinsky, B. Czerniak, R. Drake, P. Engstrom, M. Hollingsworth, A. Killary, H. Pass, J. Semmes and P. Wagner, as well as to the following for their organizational and logistics support: Drs. J. Kagan and L. Sorbara, Mr. D. Johnsey and Ms. R. Malnik. 